\title{
Percepção dos pacientes transplantados renais sobre a farmacoterapia imunossupressora: perspectivas e dificuldades
}

\author{
Perception of renal transplant patients on immunosuppressive pharmacotherapy: \\ perspectives and difficulties \\ Percepción de pacientes con trasplante renal en farmacoterapia inmunosupresora: \\ perspectivas y dificultades
}

Alan Rodrigues da Silva ${ }^{1 *}$, Flavilene Monteiro de Almeida Barbosa1, Patrícia Quirino da Costa1, Cleíse Martins Rocha', Klébia Magalhães Pereira Castello Branco², Geraldo Bezerra da Silva Junior ${ }^{3}$, Rita Mônica Borges Studart ${ }^{3}$.

\section{RESUMO}

Objetivo: Descrever a percepção dos pacientes transplantados renais sobre a farmacoterapia imunossupressora e quais suas principais dificuldades inseridas em seu contexto diário. Métodos: Trata-se de um estudo qualitativo, descritivo e exploratório. A entrevista ocorreu com auxílio de um questionário semiestruturado. Os dados foram submetidos a análise lexical no Software Iramuteq e analisados por meio da Classificação Hierárquica Descendente (CHD), Análise Fatorial por Correspondência (AFC), e por fim, foi gerada a Nuvem de palavras. Resultados: Foram entrevistados 21 pacientes, com idade entre 19 e 76 anos. O corpus textual foi separado em 448 segmentos de texto (ST), com aproveitamento de 360 ST $(80,36 \%)$. Os dados inicialmente emergiram em quatro classes e subdividias em duas ramificações do corpus total da análise: Classe $1(30,8 \%)$, Classe $2(24,4 \%)$, Classe $3(23,1 \%)$ e Classe $4(21,7 \%)$. A AFC reproduziu as classes no plano cartesiano, onde as classes 2 e 3 difundem-se nos quadrantes superiores, enquanto as classes 1 e 4 representadas em quadrantes opostos. As evocações com maior frequência ("Tomar", "Hora", "Medicação") foram consideradas e organizadas na nuvem de palavras. Conclusão: Conclui-se que os pacientes transplantados renais enfrentam dificuldades no regime imunossupressor e são susceptíveis a potenciais efeitos adversos.

Palavras-chave: Transplante renal, Imunossupressão, Sinais e sintomas.

\begin{abstract}
Objective: To describe the perception of kidney transplant patients about immunosuppressive pharmacotherapy and what are their main difficulties in their daily context. Methods: This is a qualitative, descriptive and exploratory study. The interview took place with the aid of a semi-structured questionnaire. The data were submitted to lexical analysis in the Iramuteq Software and analyzed using Descending Hierarchical Classification (CHD), Correspondence Factor Analysis (AFC), and finally, the Word Cloud was generated. Results: 21 patients were interviewed, aged between 19 and 76 years. The textual corpus was separated into 448 text segments (ST), with use of 360 ST (80.36\%). The data initially emerged in four classes and subdivided into two branches of the total corpus of the analysis: Class $1(30.8 \%)$, Class $2(24.4 \%)$, Class $3(23.1 \%)$ and Class $4(21,7 \%)$. The AFC reproduced the classes in the Cartesian plane, where classes 2 and
\end{abstract}

\footnotetext{
${ }^{1}$ Hospital Geral de Fortaleza (HGF). Fortaleza - Ceará.

${ }^{*}$ E-mail: alan_rodrigues.2010@yahoo.com.br

2 Hospital Dr. Carlos Alberto Studart Gomes. Fortaleza - Ceará.

3 Universidade de Fortaleza (UNIFOR). Fortaleza - Ceará.
} 
3 spread in the upper quadrants, while classes 1 and 4 represented in opposite quadrants. The most frequent evocations ("Take", "Hour", "Medication") were considered and organized in the word cloud. Conclusion: It is concluded that renal transplant patients face difficulties in the immunosuppressive regime and are susceptible to potential adverse effects.

Keywords: Kidney transplantation, Immunosuppression, Signs and symptoms.

\section{RESUMEN}

Objetivo: Describir la percepción de los pacientes con trasplante renal sobre la farmacoterapia inmunosupresora y cuáles son sus principales dificultades en su contexto diario. Métodos: Este es un estudio cualitativo, descriptivo y exploratorio. La entrevista tuvo lugar con la ayuda de un cuestionario semiestructurado. Los datos se sometieron a análisis léxico en el software Iramuteq y se analizaron utilizando la Clasificación jerárquica descendente (CHD), el Análisis del factor de correspondencia (AFC) y, finalmente, se generó la nube de palabras. Resultados: Se entrevistaron 21 pacientes, con edades comprendidas entre 19 y 76 años. El corpus textual se separó en 448 segmentos de texto (ST), con el uso de 360 ST (80,36\%). Los datos surgieron inicialmente en cuatro clases y se subdividieron en dos ramas del corpus total del análisis: Clase $1(30.8 \%)$, Clase $2(24.4 \%)$, Clase $3(23.1 \%)$ y Clase $4(21,7 \%)$. La AFC reprodujo las clases en el plano cartesiano, donde las clases 2 y 3 se extendieron en los cuadrantes superiores, mientras que las clases 1 y 4 se representaron en cuadrantes opuestos. Las evocaciones más frecuentes ("Tomar", "Tiempo", "Medicación") fueron consideradas y organizadas en la nube de palabras. Conclusión: Se concluye que los pacientes con trasplante renal enfrentan dificultades en el régimen inmunosupresor y son susceptibles a posibles efectos adversos.

Palabras clave: Trasplante de riñón, Inmunosupresión, Signos y síntomas.

\section{INTRODUÇÃO}

O transplante renal é a opção terapêutica de escolha para pacientes com doença renal crônica em fase terminal. Inserem-se nos grupos de maior suscetibilidade pacientes hipertensos, diabéticos, idosos, com doença cardiovascular e em uso de drogas nefrotóxicas. O Brasil é o segundo em número absoluto de transplantes renais entre 35 países, considerando o ano de 2017, com 5.929 transplantes. Os pacientes transplantados seguem com um regime imunossupressor, sendo primordial a adesão terapêutica para garantir prognóstico com resultados clínicos positivos e prevenir rejeições (YANG H, et al., 2019; ABTO, 2018).

Um dos aspectos mais relevantes para o sucesso do transplante consiste no fato de o paciente seguir orientações e recomendações da equipe especializada após o procedimento, sobretudo no que se refere ao regime medicamentoso complexo que deverá ser seguido. Para o êxito desse tipo de tratamento, devem ser incluídas também variáveis como as características demográficas, sociais e econômicas, pois tem forte influência para o desfecho clínico após o transplante (MARTINS BCC, et al.,2013).

O tratamento farmacológico, com a associação de imunossupressores e outros fármacos, requer do paciente dedicação, compreensão e capacidade para administração dos mesmos. Assim, a alternativa do transplante deve ser avaliada criteriosamente e individualmente para cada paciente, desenvolvendo critérios e estratégias singulares para pacientes com dificuldades de adesão (CHISHOLM-BURNS MA, et al., 2008; MARTINS BCC, et al., 2013).

Os imunossupressores fazendo parte do elenco terapêutico são reconhecidos por apresentarem margem de segurança estreita (índice terapêutico estreito), isto é, a dose terapêutica é próxima da dose tóxica. Somase a este problema a variabilidade dos imunossupressores, os riscos de interações medicamentosas e os efeitos adversos, enfatizando a importância da adesão terapêutica (BRASIL, 2007; SILVA DKS e ANDRADE FM, 2007). Diante desse contexto, o presente estudo justifica-se devido à complexidade e ao enorme número de informações/orientações fornecidas aos pacientes submetidos ao transplante renal durante o internamento 
e no momento da alta hospitalar, fazendo-se necessário o desenvolvimento de estratégias multiprofissionais para maior compreensão e adesão dos pacientes ao tratamento medicamentoso. O estudo objetivou descrever a percepção dos pacientes transplantados renais sobre a farmacoterapia imunossupressora e quais suas principais dificuldades inseridas em seu contexto diário.

\section{MÉTODOS}

Trata-se de um estudo exploratório e descritivo, de abordagem qualitativa. A coleta das informações e dados dos pacientes foram obtidos individualmente através de uma entrevista farmacêutica no momento oportuno da internação em um Hospital terciário de referência do Estado, no período de fevereiro a abril de 2020, com pacientes transplantados renais.

Foi utilizado a entrevista semiestruturada elaborada e validada pelos próprios pesquisadores, composta por 9 perguntas, contemplando assuntos, como: contexto diário na administração da medicação (rotina e dificuldades); sinais e sintomas e a disponibilidade dos imunossupressores ambulatorialmente. A validação do instrumento para as entrevistas seguiram as orientações de Souza AC, et al. (2017) utilizando abordagens qualitativa e quantitativa como método para a validação com os juízes, em que a qualitativa foi realizada por um grupo de especialistas na área de interesse e a quantitativa por meio de uma metodologia que mensurou o percentual de conformidade entre os juízes sobre determinado aspecto de um construto mediante o cálculo do Índice de Validade de Conteúdo (IVC).

Os depoimentos foram gravados e transcritos de forma exaustiva na tentativa de gerar indicadores qualitativos e quantitativos. Proposto por Bardin L (1976), há necessidade de caracterização das classes e posteriormente analisar o conteúdo de todos os discursos.

Foram inclusos pacientes transplantados renais há pelo menos um ano, maiores de 18 anos, que manifestassem interesse em participar do estudo e encontraram-se com estado físico e psicológico favorável para comunicar-se e responder à entrevista. Foram excluídos os pacientes com transplante duplo (pâncreasrim). Segundo Fontanella BJB, et al. (2008), o tamanho da amostra na pesquisa qualitativa se deu quando as respostas/argumentações começaram a se repetir, estabelecido pela saturação.

Os dados foram analisados por meio do software IRAMUTEQ (Interface de $R$ pour les Analyses Multimensionnelles de Textes et de Questionnaires) (SOUZA MAR, et al., 2018). Foram realizadas análises lexicográficas clássicas no Iramuteq para compreender os dados estatísticos e quantificar as evocações e formas. Obteve-se a Classificação Hierárquica Descendente (CHD) para aferir os dados do dendograma em função das classes geradas, considerando as palavras com $X^{2}>3,84(p<0,05)$. Posteriormente, realizada a Análise Fatorial por Correspondência (AFC). Por fim, foi gerado a Nuvem de Palavras, que unifica as palavras e dispõem graficamente em função da sua frequência.

O estudo foi submetido à apreciação do comitê de ética e pesquisa (CEP) do Hospital Geral de Fortaleza, o qual foi avaliado sua viabilidade de sua realização e sendo aprovado sob o número de parecer 3.934.154. $O$ estudo respeitou todas as etapas das diretrizes e normas de pesquisa envolvendo seres humanos da Resolução 466/12 do Conselho Nacional de Saúde (BRASIL, 2012). Foi requerido aos participantes a leitura e assinatura do Termo de Consentimento Livre Esclarecido (TCLE).

\section{RESULTADOS}

Foram entrevistados 21 pacientes, com idade entre 19 e 76 anos, sendo $62 \%$ do sexo masculino e $38 \%$ do sexo feminino. Em relação à escolaridade, $9 \%$ possuíam ensino fundamental incompleto, $67 \%$ com ensino médio e $24 \%$ com ensino superior.

Quanto ao tempo de transplante, $67 \%$ dos pacientes já tinham mais de um ano e $33 \%$ dos pacientes mais de dois anos de transplante. As análises dos dados qualitativos foram realizadas com desígnio de compreender o processo de terapia farmacológica que engloba perspectivas e dificuldades nos pacientes transplantados renais. O corpus geral foi constituído por 21 textos, separados em 448 segmentos de texto 
(ST), com aproveitamento de 360 ST (80,36\%). Emergiram 15.739 ocorrências (palavras, formas ou vocábulos), sendo 1.612 palavras distintas e $758 \mathrm{com}$ uma única ocorrência. O conteúdo analisado foi categorizado em quatro classes: Classe 1, com 111 ST (30,83\%); Classe 2, com 88 ST (24,44\%); Classe 3 , com 83 ST (23,06\%); e Classe 4, com 78 ST (21,67\%).

Enfatiza-se que essas quatro classes se encontram divididas em duas ramificações ( $A$ e $B$ ) do corpus total em análise. O subcorpus $A$, recebe o nome de sua única Classe 1 ("Rotina diária e o enfrentamento a terapia imunossupressora"). O subcorpus B, denominado "Dificuldades no percurso terapêutico", que contém os discursos correspondente à Classe 2 ("Dificuldades na administração dos medicamentos"), Classe 3 ("Dificuldades diante a disponibilidade dos imunossupressores") e classe 4 ("Sinais e Sintomas") (Figura 1).

Figura 1 - Dendograma da Classificação Hierárquica Descendente.

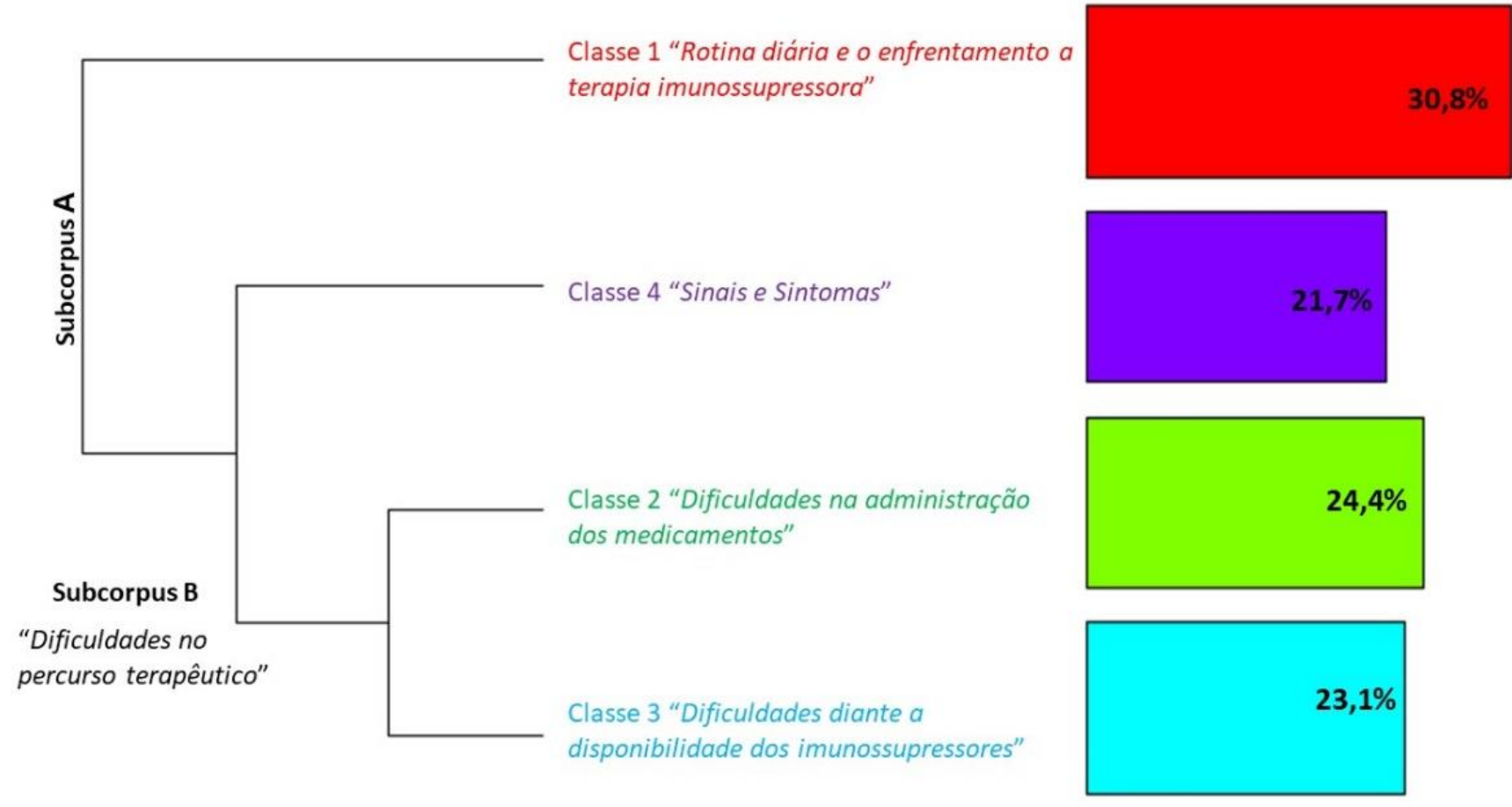

Fonte: Silva AR, et al., 2020.

Com o intuito de melhor ilustrar as palavras do corpus textual em suas referentes classes, organizou-se um diagrama com exemplos de palavras de cada classe avaliadas por meio do teste qui-quadrado $\left(X^{2}\right)$. Nele emergem as evocações que apresentam vocabulário semelhante entre si e vocabulário diferente das outras classes. Em seguida serão apresentadas, operacionalizadas e exemplificadas cada uma dessas classes encontradas por meio da análise de Classificação Hierárquica Descendente (Figura 2). 
Figura 2 - Organograma de classes a partir das entrevistas com pacientes transplantados renais.

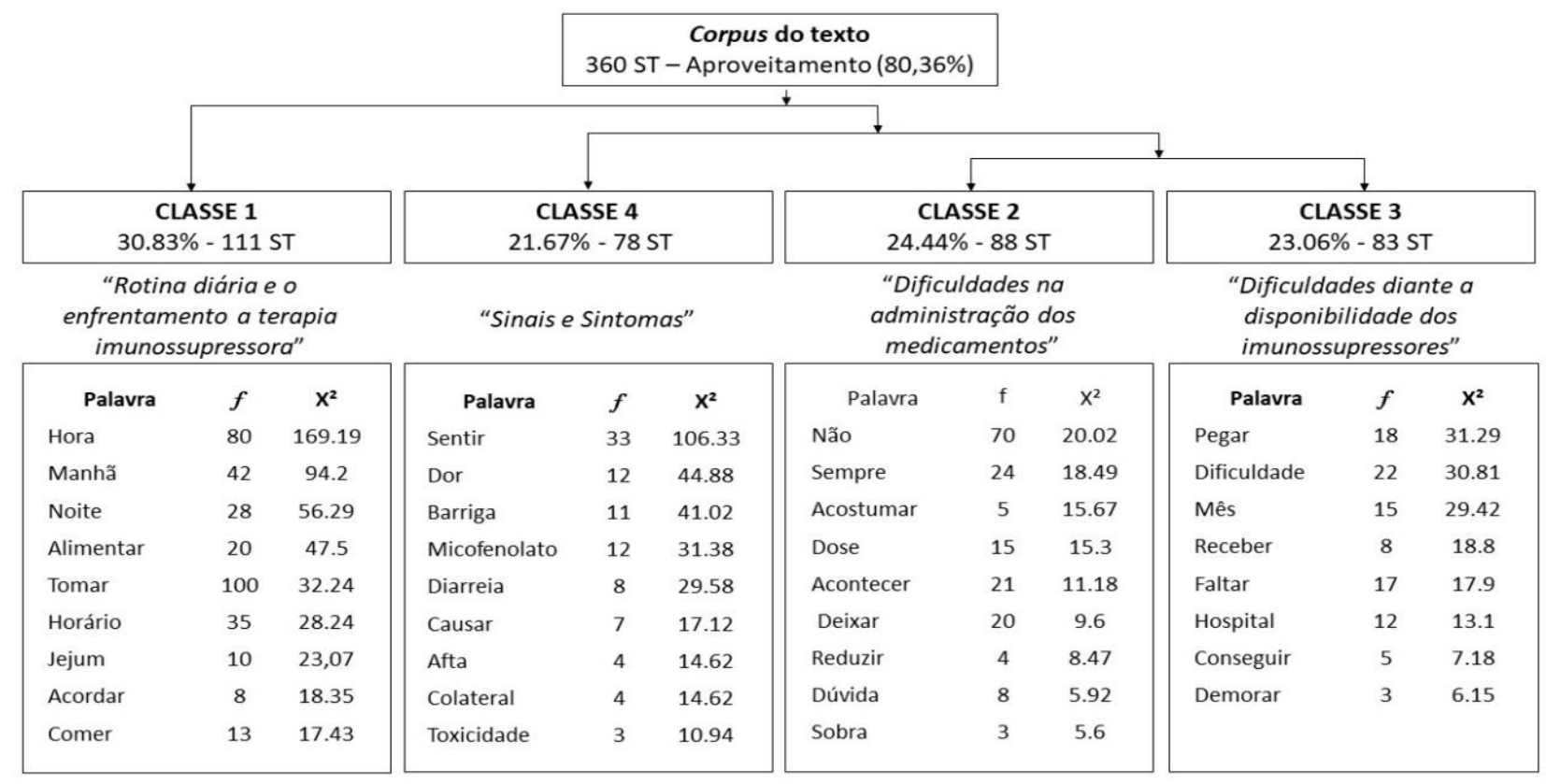

Fonte: Silva AR, et al., 2020.

\section{Classificação Hierárquica Descendente}

\section{Classe 1 - Rotina diária e o enfrentamento a terapia imunossupressora}

Compreende $30.83 \%$ ( $f=111 \mathrm{ST})$ do corpus total analisado. Essa classe é constituída por palavras e radicais no intervalo entre $X^{2}=3.85$ (Sangue) e $X^{2}=169.19$ (Hora). Essa classe traz conteúdo referente à rotina dos pacientes diante seus hábitos em relação aos horários pré-estabelecidos para administração dos medicamentos. As inúmeras informações fornecidas pela equipe, transcende ao paciente aglomerado de atividades a serem inseridas dentro do cotidiano. Evidencia-se, a partir da análise da classe, que há dúvidas sobre a orientação de determinados imunossupressores interagirem com a alimentação. Diante desse contexto, outros relatos demonstram fragilidade no armazenamento correto e também como utilizá-los diariamente fora do domicílio.

"Eu fiz o kit do rim, que tem um vidrinho, que tem o nome "kit do rim", separo à noite para tomar de manhã, como à noite eu tomo só os tacrolimos, já separo antes." E4

Eu não sei o porquê desse negócio da alimentação, outros medicamentos que tem que tomar na hora do café, então é confuso, por que tem que fazer isso só com os imunossupressores. E9

"[...] quando saio de casa eu levo um porta-comprimido e eu coloco dentro dele, eu tiro da cartelinha e coloco dentro separado tacrolimos, sirolimos e os remédios da pressão que tomo durante o dia." E17

O retorno às atividades diárias aparentemente é um ganho relatado por diversos pacientes ao saírem do processo de hemodiálise, em contrapartida o transplante renal dispõe para o paciente maior liberdade para desfrutar de prazeres simples como ter uma alimentação com menor restrição, beneficia melhor interação social e autonomia para realizar atividades do cotidiano.

Por outro lado, os hábitos do paciente transplantado e o processo diário ligado a medicação é relatado com uma grande carga de tensão e observado como um forte estressor a estes pacientes, destaca-se nesse contexto que desempenhar o seguimento rotineiro de horários complexos e fragmentados na administração dos fármacos e com a alimentação interfere negativamente a comodidade e o bem-estar do paciente (BRITO DCS, et al., 2015; NEIPP M, et al., 2009). 
Segundo os dados do estudo, corrobora que episódios de estresse moderado a intenso e as preocupações com horários, rotina e hábitos a serem seguidos podem acompanhar os pacientes desde o período pós transplante imediato até 4 anos, após esse período os pacientes relatam melhor adaptação mantendo assim melhor qualidade vida em sua rotina.

Diante desse contexto, a aceitabilidade e aderir ao novo estilo de vida é um processo progressivo (CHEN KH, et al., 2010; FALLON M, et al., 1997). Perpassa a rotina de muitos pacientes a preocupação constante da rejeição e há necessidade de incluir nos hábitos diários a adesão de regimes complexos da medicação, neste caso, engloba administração correta e o seguimento de horários posológicos, além disso o armazenamento e a preservação dos medicamentos garantindo a viabilidade e a estabilidade dos mesmos (SANTOS LF, et al., 2018; BRITO DCS, et al., 2015).

\section{Classe 2 - Dificuldades na administração dos medicamentos}

Foi constituída por $24.44 \%$ ( $f=88 \mathrm{ST}$ ) do corpus total analisado. Composta por palavras e radicais no intervalo entre $X^{2}=4.13$ (Buscar) e $X^{2}=30.37$ (Jeito).

Verificou-se pontualmente as principais dificuldades na administração dos imunossupressores, soma-se a isto a mudança constantemente da posologia e da classe terapêutica, demonstrando-se nos relatos a dificuldade no entendimento e no seguimento diário da medicação.

Relataram ainda sobre a problemática do esquecimento na administração devido horários não estratégicos, em consequência de pacientes ativos em formas de trabalhos distintos.

"[...] dúvida: eu tomava sirolimos, aí meu médico trocou por micofenolato, no caso faltando o myfortic na farmácia, posso tomar o sirolimos para não ficar sem a medicação? [...] pra eu não ficar sem, eu tomei a sobra do sirolimos." E1

"Aconteceu semana passada de em vez de eu tomar um micofenolato, eu tava tomando dois, confundi com a dose do tacrolimos. E ia faltar medicamento se eu não tivesse ido buscar [...]" E5

O esquecimento foi um gatilho para o fracasso terapêutico relatada por muitos pacientes, em alguns casos levou a instabilidade no sucesso do transplante. Outrossim, os entrevistados revelam a necessidade de estratégias para aderir a medicação de forma mais segura.

"Se for por mim eu não tomo não, eu esqueço, eu tomo nenhum, eu nunca lembro, minha filha que sempre está no meu pé." E5

"Meus rins estavam parando por que eu não estava tomando os remédios direito [...] vou voltar pra hemodiálise, eu perdi o transplante, mas ainda continuo fazendo xixi." E19

Determinar horários distante da realidade do paciente é uma condição que favorece a não adesão medicamentosa e dificulta o curso terapêutico. Sobre esse aspecto, frisam que os horários são fragmentados durante o dia e que prejudicam a realização de atividades simples do cotidiano.

"Como trabalho, já aconteceu de estar no trabalho, chegar o horário e está sem medicação para tomar na hora." E13

"No começo esses horários são bastante chatos, acordar muito cedo só pra tomar esses remédios [...] já teve caso de acordar sonolento, tomar a medicação, e ficar na dúvida se tomei ou não." E18

$\mathrm{Na}$ classe 2 destacam-se as dificuldades frente a imunossupressão e a ocorrência de não adesão. $\mathrm{O}$ estudo de Klewitz F, et al. (2019), ressalva a postura do paciente transplantado renal na qual demonstram interesse nas informações sobre os medicamentos, por outro lado, tais orientações fornecidas são completa e de natureza complexa o que dificulta o entendimento do paciente, diante disso, percebe-se a insatisfação dos pacientes frente as recomendações terapêuticas. Por essa razão, a partir dos relatos observa-se 
dificuldade no entendimento de doses e principalmente na ocorrência de alterações nas classes farmacológicas.

O maior risco da não adesão é devido a elevada quantidade de medicamentos para uso diário. Soma-se a isto, a dificuldade para recordar com precisão a medicação no horário estabelecido, corrobora-se com a problemática do esquecimento apresentado nos relatos, possibilitando a ocorrência de potencializar o efeito imunossupressor em casos específicos ou proporcionar falha terapêutica (LOW JK, et al., 2019; MORRISSEY PE, 2007).

\section{Classe 3 - Dificuldades diante a disponibilidade dos imunossupressores}

Representa $23.06 \%(\mathrm{f}=83 \mathrm{ST})$ do corpus total analisado. Composta por palavras e radicais no intervalo de $X^{2}=3.9$ ("Fortaleza") e $X^{2}=31.29$ ("Pegar"). Emergiram diversos relatos frente a um problema que é discutido de forma nacional, a indisponibilidade de medicamentos no Componente Especializado do Sistema Único de Saúde. Além disso, os entrevistados expuseram os medos e as incertezas diante da falta dos medicamentos. Em contrapartida, surgiram um conjunto de questões que dificulta o acesso ao medicamento.

"Faltou várias vezes, não foi nem uma, nem duas, foram várias vezes de minha esposa ir pegar remédio e não ter. Várias vezes chegou aí e não tinha imunossupressor." E10

"Já tomei uma dose menor por que não tinha a medicação na farmácia, eu diminui para dar mais tempo no mês [...] eu fiz isso por que estava faltando né." E14

"Eu sinto dificuldade quando é pra renovar a receita por que demora demais, por que tem que passar pelo farmacêutico, mas depois é mais rápido." E17

"Algumas vezes já faltou medicamento aí eu não sabia, fiquei vários dias sem tomar. Todo dia eu ia no hospital saber se conseguia, mas chegou um ponto que até fiquei internada por que já estava passando mal sem a medicação." E19

O Brasil possui o Componente Especializado da Assistência Farmacêutica, que em sua importância significativa destaca a dispensação de medicamentos de alto custo, incluindo-se os imunossupressores. Vale ressaltar que a dispensação dos imunossupressores é gratuita, e o acesso deve ser garantido pelo Sistema Único de Saúde (SANTOS BP, et al., 2017; ARRUDA GO e RENOVATO RS, 2012). Inclui o risco e a incerteza de conviver com a falta desses medicamentos que dito essenciais para o sucesso do transplante, integra-se a essa problemática o elevado risco de rejeição do órgão transplantado (ALMEIDA DES, et al., 2013; OLIVEIRA M et al., 2014).

A indisponibilidade e escassez na dispensação dos imunossupressores consta como um problema de vários pacientes, onde buscam estratégias para minimizar a falta e garantir o acesso. Destaca-se o contato constante com as equipes de transplante e esforço em manter contato com outros estados que possam suprir a falta. Infere-se que compete as organizações e a programação assistencial garantirem o acesso aos medicamentos essenciais para manutenção do transplante (ALMEIDA DES, et al., 2013; ARRUDA GO e RENOVATO RS, 2012).

\section{Classe 4 - Sinais e Sintomas}

Representa $21.67 \%$ ( $f=78 \mathrm{ST}$ ) do corpus total analisado. Composta por palavras e radicais no intervalo de $X^{2}=3.85$ ("Diminuir") e $X^{2}=106.33$ ("Sentir"). Os entrevistados relataram sobre as consequências e os eventos adversos no tratamento proposto. Emergiram-se falas dos principais efeitos que o organismo sofre devido ao uso contínuo dos imunossupressores e os desafios para o controle e a minimização deles. Relatam ainda incertezas e dúvidas diante sintomas intensos e graves.

"Às vezes eu sinto enjoo e as vezes queimação. Não é sempre não, mas as vezes eu sinto. Eu acredito que é do medicamento devido eu tomar muitos né." E1 
"Eu sei que veio essa diabetes por causa desse remédio, por que quando eu fazia hemodiálise [...] eu não tinha outra doença." E8

"Uso micofenolato, eu comecei a ter muita afta e uma suadeira muito forte, suor em excesso [...] um cheiro estranho no corpo, sei lá, um cheiro desagradável [...] eu mesmo diminui a dose e melhorei do suor e as aftas diminuíram." E9

"Quando eu tomo o remédio com pouco tempo vou no banheiro, eu sinto muita diarreia e dores de barriga." E19

Estudos mostram que os eventos adversos provedores dos medicamentos imunossupressores é um aspecto fundamental para fragilizar a qualidade de vida dos pacientes. Emerge nesse contexto que o paciente pós transplante necessita aderir precisamente ao regime terapêutico para o resto da vida, consequentemente, são predispostos ao risco de infecções e reações negativas aos medicamentos (CHEN KH, et al., 2010; ADRIANO LS, et al., 2017).

Fatores determinantes a não adesão em pacientes adultos transplantados renais refere que a complexidade do regime imunossupressor e os relatos subjetivos dos pacientes após uso dos mesmos e a ocorrência sinais e sintomas que os fazem por decisão particular a suspensão ou a modificação da condução terapêutica. Necessário a monitorização e a supervisão contínua para avaliar o limiar entre a dose terapêutica e a toxicidade (DENHAERYNCK K, et al., 2005; MORRISSEY PE, et al., 2007).

Em um estudo foi possível avaliar os efeitos dos imunossupressores corrobora-se com os relatos do nosso estudo, ocorreram desde fragilidade na pele, diarreia, edemas faciais, fraqueza muscular até mal estar, certifica que os pacientes ao sentirem tais efeitos são acometidos por estresse, causando impacto negativo na qualidade de vida. À vista disso, destaca-se a prevalência de uso nos esteroides, ciclosporina, micofenolato, azatioprina e tacrolimos (ROSENBERGER J, et al., 2005; RAVAGNANI LMB, et al., 2007).

\section{Análise Fatorial por Correspondência}

Por meio da AFC, foi possível ainda realizar comparações das diferenças de evocações, independente das classes as quais pertencem, considerando a frequência de incidência de palavras e as classes, representando-as em um plano cartesiano.

Observa-se que as palavras das classes 2 e 3, difundem-se, e estão mais próximas entre si, tais como "Dificuldade", "Risco", "Acostumar", "Receber", "Faltar", "Farmácia" e "Pegar", assim assemelham-se nos assuntos propostos pelas classes, no entanto, apresenta-se afastada das demais. As classes 1 e 4 estão opostas entre as classes já citadas e em quadrantes distintos (Figura 3).

Considerando a disposição das classes e aquilo que elas representam, é pertinente classificar os eixos do plano cartesiano para uma melhor compreensão da disposição das classes no mesmo. Desse modo, considera-se que o Eixo $\mathrm{Y}$, precisamente localizados nos quadrantes superiores, é o eixo que emerge assuntos acerca das dificuldades na administração dos medicamentos e o acesso aos imunossupressores.

Segundo Xu XF, et al. (2018), o paciente pós transplante possui variabilidade em esquemas terapêuticos, estando assim vulneráveis a ajustes constantes de doses e predisposto potenciais eventos adversos, além do risco da indisponibilidade dos medicamentos.

Já no Eixo X, é visivelmente distinto em duas classes, localizadas quadrantes opostos, envolvendo assuntos diferentes. Assim, os dois quadrantes inferiores dispõem temáticas referente as classes 1 e 4 . No quadrante inferior esquerdo, engloba assuntos que se refere a rotina e hábitos dos pacientes no processo de adequação ao regime imunossupressor, composto majoritariamente pela classe 1, ("hora", "manhã", "comer", "noite", "alimentar").

Por fim, o quadrante inferior direito, onde predomina a classe 4, inclui questões acerca dos principais efeitos adversos provocados pelo uso continuo dos imunossupressores, que diante dos relatos interfere negativamente no processo de adesão (“Sentir”, "Corpo", “Toxicidade”, “Diarreia”, "Dor”). 
Figura 3 - Análise Fatorial por Correspondência.

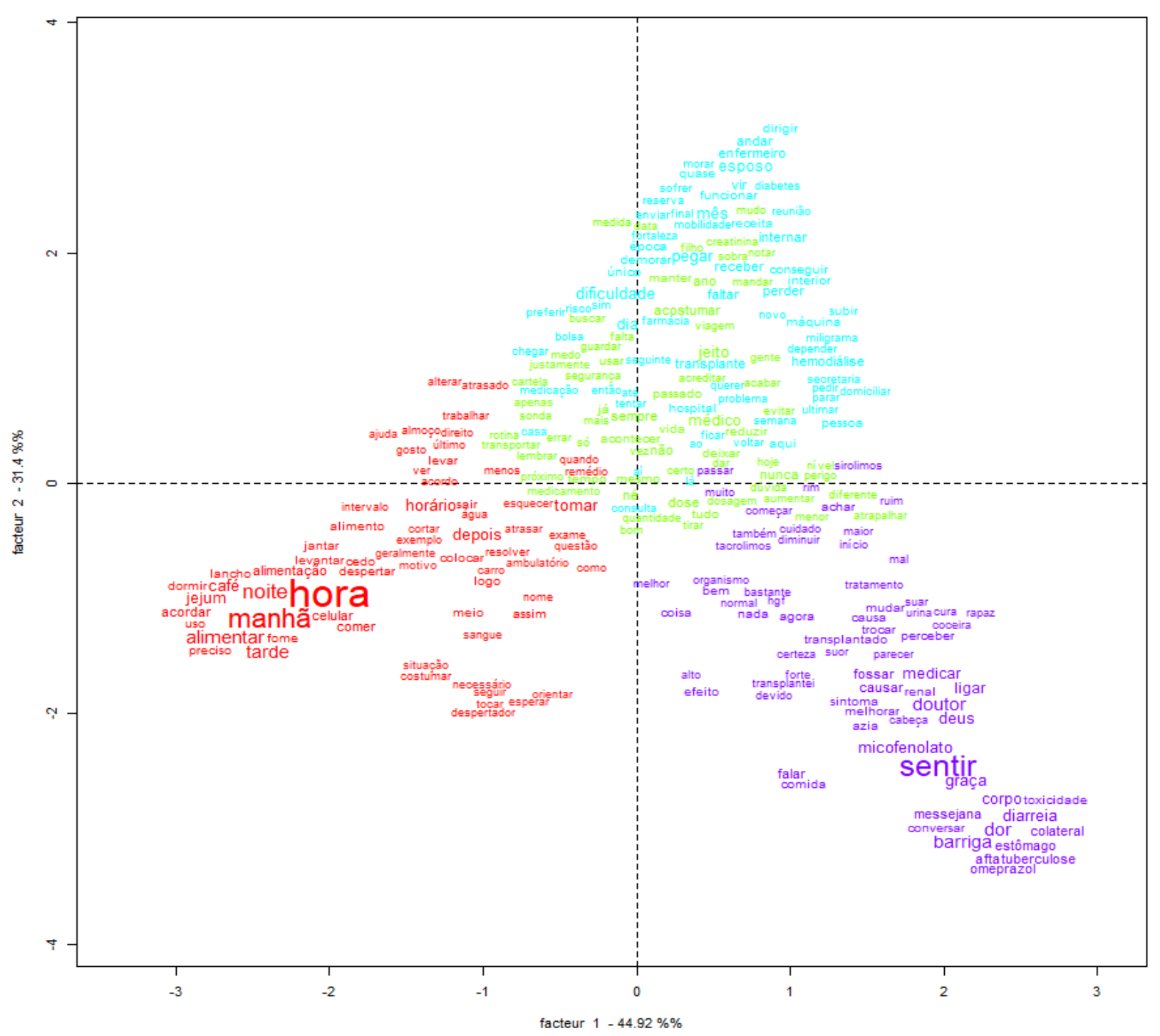

Fonte: Silva AR, et al., 2020.

\section{Nuvem de palavras}

Em seguida, foi analisada a nuvem de palavras obtida por meio dos discursos dos próprios participantes, verifica-se que as palavras mais evocadas foram: "Tomar" ( $f=539)$, "Hora" ( $(=219)$, "Medicação" $(f=132)$, "Remédio" ( $f=113)$, "Manhã" ( $(=101)$, "Sempre" ( $f=77)$, "Sentir" ( $f=61)$, "Esquecer" ( $(=58)$, "Faltar" ( $f=43)$, "Dose" ( $f=32)$, "Alimentar" ( $f=26)$, "Guardar" ( $f=26)$ e "Dúvida" ( $f=24)$. (Figura 4). 
Figura 4 - Nuvem de palavras.

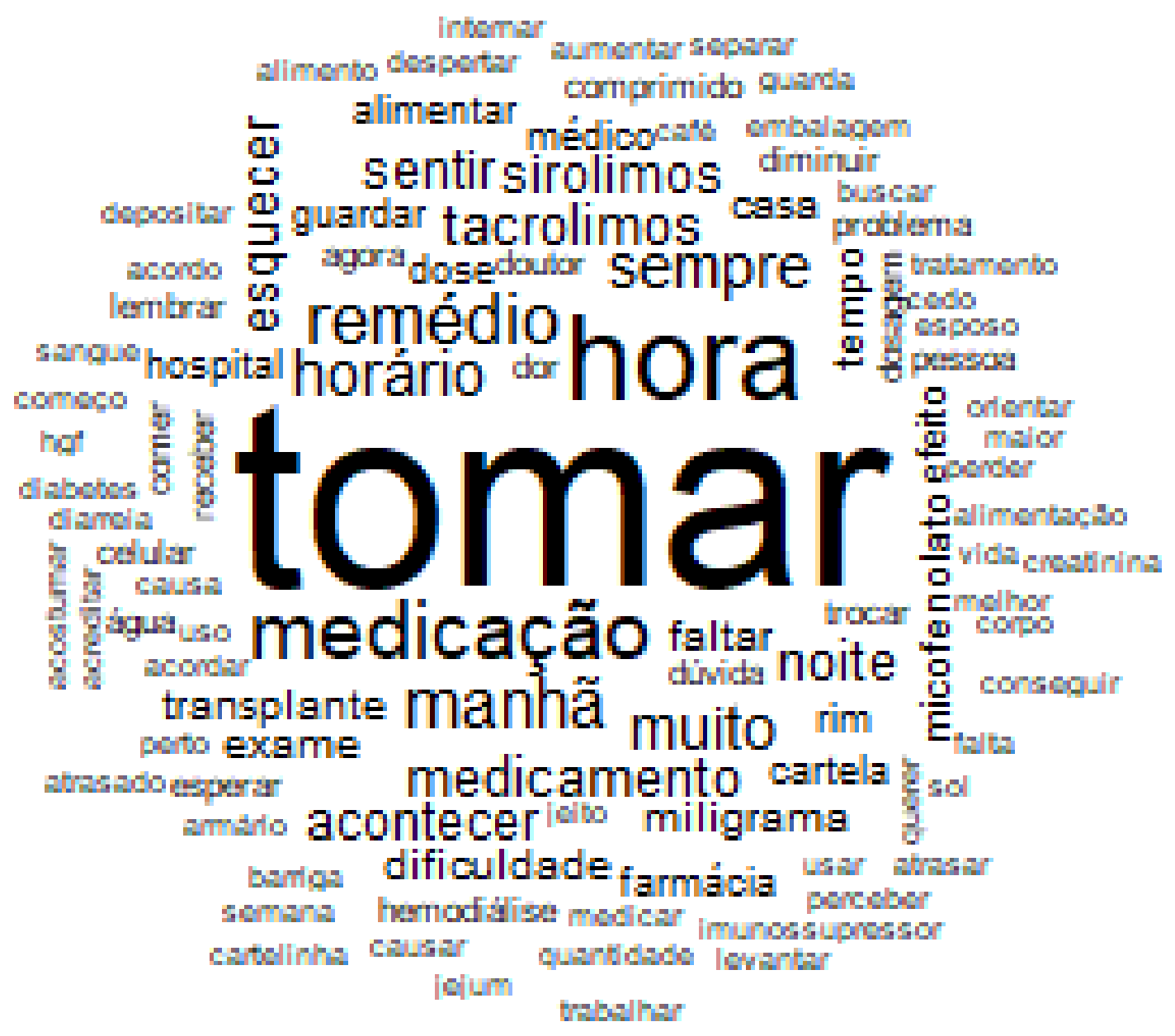

Fonte: Silva AR, et al., 2020.

A rotina, alimentação e a medicação são os pilares para o sucesso do transplante renal, que perpassa por todas as dificuldades de enfrentamento na disponibilidade dos medicamentos, além de horários complexos, dúvidas e incertezas (ALLOWAY RR, et al., 2011). Fundamenta-se a partir dos relatos dos entrevistados que há questionamentos quanto ao sentirem sintomas que julgam estarem relacionado com os imunossupressores. São evidenciados na imagem acima e por meio das evocações com maior frequência, demonstrado através dos fármacos responsáveis e pelos efeitos negativos deles.

\section{CONCLUSÃO}

Ressalta que por meio dos dados gerados na pesquisa e no contexto em que estão inseridos, o paciente pós transplante renal é submetido copiosas informações que exigem do mesmo seguir com plenitude e diligência todas as recomendações para o sucesso terapêutico e que acompanhe os resultados positivos do transplante. Desse modo, torna-se primordial atuação conjunta e multiprofissional ao paciente transplantado renal, destacando-se as atribuições clínicas do farmacêutico em propor horários favoráveis e convenientes, fazendo assim que haja melhor adaptação ao regime terapêutico e proporcionando melhor adesão, em contrapartida conhecendo e amenizando os efeitos negativo dos fármacos e oportunizando-os melhor qualidade de vida. 


\section{REFERÊNCIAS}

1. ADRIANO LS, et al. Pharmaceutical interventions and their clinical outcomes in an inpatient post-transplant unit. Rev. Bras. Farm. Hosp. Fortaleza, 2017; 8(1): 15-21.

2. ALLOWAY RR, et al. Evolution of the Role of the Transplant Pharmacist on the Multidisciplinary Transplant Team. American Journal of Transplantation, Carolina, 2011; 11(1): 1576-1583.

3. ALMEIDA DES, et al. Normative evaluation of the immunosuppressive drug prescription and dispensing process for renal transplant patients in the state of Minas Gerais, Brazil, 2008. Epidemiol. Serv. Saúde, Brasília. 2013; 22(4): 651-660.

4. ARRUDA GO, RENOVATO RD. Uso de medicamentos em transplantados renais: práticas de medicação e representações. Rev Gaúcha Enferm, 2012; 33(4): 157-164.

5. ASSOCIAÇÃO BRASILEIRA DE TRANSPLANTE DE ÓRGÃOS. Dimensionamento dos Transplantes no Brasil e em cada estado. São Paulo: ABTO, 2018; 89p.

6. BARDIN L. Análise de Conteúdo. France: Edições 70, 1979; 229p.

7. BRASIL. Ministério da Saúde. Conselho Nacional de Saúde. Resolução n.354/07. Dispõe sobre Boas Práticas de Manipulação de Preparações Magistrais e Oficinais para Uso Humano em farmácias. Brasília: Agência Nacional de Vigilância Sanitária, 2007.

8. BRASIL. Ministério da Saúde. Conselho Nacional de Saúde. Resolução n.466/12. Dispõe sobre diretrizes e normas regulamentadoras de pesquisas envolvendo seres humanos. Brasília: Conselho Nacional de Saúde, 2012.

9. BRITO DCS, et al. Análise das mudanças e dificuldades advindas após o transplante renal: uma pesquisa qualitativa. Rev. Latino-Am. Enfermagem, 2015; 23(3): 419-426.

10. CHEN KH, et al. Stress and stress-related factors of patients after renal transplantation in Taiwan: a cross-sectional study. Journal of Clinical Nursing. Taichung, 2010; 19(1): 2539-2547.

11. CHISHOLM-BURNS MA, et al. Impact of clinical pharmacy services on renal transplant recipients' adherence and outcomes. Dove Medical Press Ltd. Tucson, 2008; 2: 287-292.

12. DENHAERYNCK K, et al. Prevalence, consequences, and determinants of nonadherence in adult renal transplant patients: a literature review. Transplant International. Switzerland, 2005; 18: 1121-1133.

13. FALLON M, et al. Stress and quality of life in the renal transplant patient: a preliminary investigation. Journal of Advanced Nursing. London, 1997; 25: 562-570.

14. FONTANELLA BJB, et al. Saturation sampling in qualitative health research: theoretical contributions. Cad. Saúde Pública, 2008; 24: 17-27.

15. KLEWITZ F, et al. Information Needs of Patients About Immunosuppressive Medication in a German Kidney Transplant Sample: Prevalence and Correlates. Frontiers in Psychiatry. Hannover, 2019; 10: 1-13.

16. LOW JK, et al. Improving medication adherence in adult kidney transplantation (IMAKT): A pilot randomised controlled trial. Scientific Reports(Nature). Victoria, 2019; 9: 1-8.

17. MARTINS BCC, et al. Pharmaceutical care in transplant patients in a university hospital: pharmaceutical interventions. Brazilian Journal of Pharmaceutical Sciences, 2013; 49(4): 659-666.

18. MORRISSEY PE, et al. Medication Noncompliance and its Implications in Transplant Recipients. Adis Data Information BV, Island. 2007; 67(10): 1463-1481.

19. NEIPP M, et al. Renal transplantation today. Langenbecks Arch Surg. Germany, 2009; 394: 1-16.

20. OLIVEIRA M, et al. Evolution of the National Policy of Care for the Person with a Kidney Disease in Brazil (1999 to 2004). Health \& Social Change, 2014; 5(3): 105-112.

21. RAVAGNANI LMB, et al. Qualidade de vida e estratégias de enfrentamento em pacientes submetidos a transplante renal. Estudos de Psicologia. Rio Preto, 2007; 12(2): 177-184.

22. ROSENBERGER J, et al. Factors modifying stress from adverse effects of immunosuppressive medication in kidney transplant recipients. Clinical Transplantation. Kosice, 2005; 9(1): 70-76.

23. SANTOS BP, et al. Utilization of immunosuppressants by people with renal transplant. J. res.: fundam. care. Online. Santos, 2017; 9(4): 1145-1153.

24. SANTOS LF, et al. Qualidade de Vida em Transplantados Renais. Psicologia USF. Bragança Paulista, 2018; 23(1): 163-172.

25. SILVA DKS, ANDRADE FM. Pharmacogenetics of selective serotonine reuptake inhibitors: a review. Nova Hamburgo, 2007; 16(7): 498-503.

26. SOUZA AC, et al. Propriedades psicométricas na avaliação de instrumentos: avaliação da confiabilidade e da validade. Epideiol. Serv. Saúde, Brasília, 2017; 26(3): 649-659.

27. SOUZA MAR, et al. The use of IRAMUTEQ software for data analysis in qualitative research. Revista da Escola de Enfermagem. Paraná, 2018; 53: 1-7.

28. XU XF, et al. Pharmaceutical Care in Kidney Transplant Recipients: Behavioral and Physiologic Outcomes at 12 Months. Transplantation Proceedings. Tainan, 2018; 50: 2451-2456.

29. YANG H, et al. Impact of pharmacist-led post-transplant medication management for kidney transplant recipients: A retrospective pre- and post-intervention study. Journal Clinical Pharmacy an Therapeutics. China, 2019; $19(1): 1-8$. 